\title{
An active stabilizer for cable-driven parallel robot vibration damping
}

\author{
Maximilian Lesellier ${ }^{1}{ }^{2}$, Loic Cuvillon ${ }^{2}$, Jacques Gangloff ${ }^{2}$ and Marc Gouttefarde ${ }^{1}$
}

\begin{abstract}
Cable-Driven Parallel Robots (CDPRs) can execute fast motions across a large workspace. However, these performances are reached at the cost of a relatively low stiffness which often yields parasitic vibrations at the CDPR mobile platform. In this paper, vibration damping of CDPRs is addressed by means of an original active stabilizer consisting of actuated rotating arms installed on-board the CDPR mobile platform. A control strategy for the whole system, which consists of the CDPR and the stabilizer, and with one purpose for eachposition control for the platform and vibration damping for the stabilizer-is designed. The system being controlled at two different time scales, the singular perturbation theory can be used to prove the stability of the corresponding closed-loop system. The efficiency of the proposed device and control strategy is tested in simulations in the case of a planar 3-DOF CDPR equipped with a three-arm stabilizer.
\end{abstract}

\section{INTRODUCTION}

A Cable-Driven Parallel Robot (CDPR) is a type of parallel robot whose mobile platform is driven by cables. Controlling the lengths of the cables by means of winches, the platform can be brought to a desired position and orientation. Using cables instead of rigid links, CDPRs can notably have a large payload-to-weight ratio, a very large workspace, high dynamics, and reconfiguration capabilities e.g. [1]-[6].

However, the advantages of flexible links is at the cost of a relatively low stiffness and, therefore, of vibrations at the CDPR mobile platform [7], [8]. Vibrations are an issue in applications requiring good positioning accuracy, low settling time, and limited fluctuations around the end-effector desired trajectory. Moreover, flexible cables undergo a deflection due to their own weight. This cable sagging may however be neglected in the case of a high payload to cable mass ratio, e.g. [9]. The cables can then be modeled as taught strings. Since the axial flexibility of the cables contributes to the mobile platform vibrations significantly more than their transversal flexibility [10], the cables are usually modeled as linear axial springs. Modeling the cables as springs instead of inextensible segments can improve control algorithm stability [11].

Vibrations damping is an active field in mechanical engineering. The damping can be either passive or active. Passive damping can only damp one specific natural frequency, and in the case of a moving CDPR platform, the vibration natural frequencies are higly dependent on the platform position and orientation [10], [12]. Active damping consists in creating an internal wrench to compensate vibrations [13]. It has already been used for CDPRs, e.g. in [1] where the stiffness of the

\footnotetext{
${ }^{1}$ LIRMM University of Montpellier, CNRS, Montpellier France

2 Icube Laboratory, University of Strasbourg, France
}

fully-constrained CDPR FALCON was increased by creating internal forces. There are two major ways to perform active vibration damping, either using input shaping methods or generating a transient wrench to compensate the vibration. In [14], an input shaping method in modal space is used to reject vibrations on an over-constrained CDPR. Using input shaping methods on a CDPR requires the winches to wind the cables fast enough. In the case of large-dimension heavypayload CDPRs, this requirement cannot always be fulfilled.

Another solution to generate an internal wrench consists in adding additional actuators [15], [16]. These actuators are directly embedded in the mobile platform and can be utilized to reduce the vibrations. Examples of such additional actuators are reaction wheels used on a CDPR in [9], Control Moment Gyros (CMG) or sliding-mass actuators [17]. However, reaction wheels and CMG can only generate a torque while linear actuators can only generate a force. They may thus not be sufficient to compensate the vibrations of the CDPR mobile platform, which act along all the Degrees of Freedom (DOF) [10].

Besides, in [18], actuated rotating arms are used to adjust the position of the center of mass of a static 6-DOF nonactuated platform. The same type of actuator can be used to generate inertial forces for dynamic reorientation as in [19]. Several can be coupled together to generate forces and torques along more than one directions [20], allowing more complex control strategies. Force and moment generation using rotating arms can thus be an interesting means to actively damp vibrations of a CDPR mobile platform. This type of method has already been used on a planar CDPR in [21] where one rotating arm is used to control the platform moment of inertia, and in [22] where two actuated rotating arms are used to provide a reaction wrench for eliminating out-of-plane vibrations occurring in the noncontrollable DOF. In these works, the platform positioning and the vibration damping are two independent tasks realized by two independent controllers.

Hereafter, a group of actuators mounted on the CDPR platform for stabilization, is called the stabilizer. The system consisting of a CDPR and its stabilizer is conceptually similar to the micro-macro approach, where a small robot is placed on a larger one, also for stabilization purposes [15], [16], [23], [24]. The two systems work on two different tasks and are controlled at two different time scales. An approach that can be used to analyze the stability of the whole system is the singular perturbation theory [25]. The latter considers that the system variables work on two different time scales, a slow one and a fast one, and thus can be decomposed as two subsystems, one for each time scale. According to this 

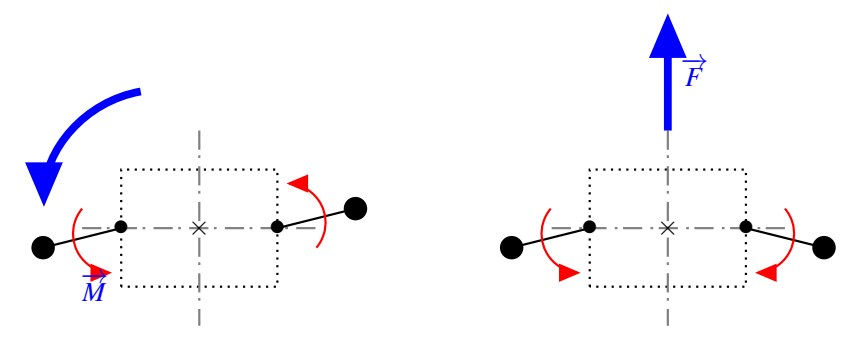

Fig. 1. Principle of the wrench generation with actuated arms

theory, the system is treated as the sum of the fast and slow subsystems, and it is stable if both subsystems are [26].

Besides vibration issues, the mobile platform might also encounter positioning errors inherent to robots in general. Various control approaches deal with this problem, including the computed-torque method, which has already been used on cable robots [27], [28].

The contribution of this paper is a multi-DOF active stabilizer consisting of rotating arms placed on-board the CDPR mobile platform to damp vibrations along all its DOFs. Since the actions of the platform computed torque position control and the stabilizer vibration damping happen at two different time scales but interact, an overall control strategy is proposed. Moreover, the stability of the corresponding control law is proved by means of the singular perturbation theory. The efficiency and robustness of the proposed control strategy is tested in simulations on a planar 3-DOF CDPR equipped with a three-arm stabilizer. Note that, unlike in [22], the stabilizer is used to compensate vibrations on all the DOFs of the CDPR platform, including the DOFs that are also actuated through the cables. Its aim is then not to work on uncontrollable DOFs but to speed up the damping on controllable ones, working together with the platform position control.

The paper is organized as follows. First, the main principle of the stabilizer is described in Section II. The dynamic model of a CDPR with an on-board stabilizer is presented in Section III. The control strategy and the proof of its stability are detailed in Section IV. A validation of the approach based on computer simulations of a planar CDPR is presented in Section V. Finally, conclusions are given in Section VI.

\section{DESIGN OF THE STABILIZER}

The main principle of a multi-DOF stabilizer consisting of several arms is illustrated in Fig. 1 in the case of two arms. Since a movement of one arm creates a wrench at the center of mass of the stabilizer, a coordinated movement of the two arms can create a single force or a single moment, the unwanted components of the wrench of one arm being compensated by the other arm. In the simple two-arm twoDOF case shown in Fig. 1, two symmetric arms create a moment by moving in the same direction and a force by moving in opposite directions. With this principle and using a number of stabilizer arms equal to or greater than the number of mobile platform DOFs, it is possible to generate wrenches along all the DOFs, as long as no pair of arms are aligned.

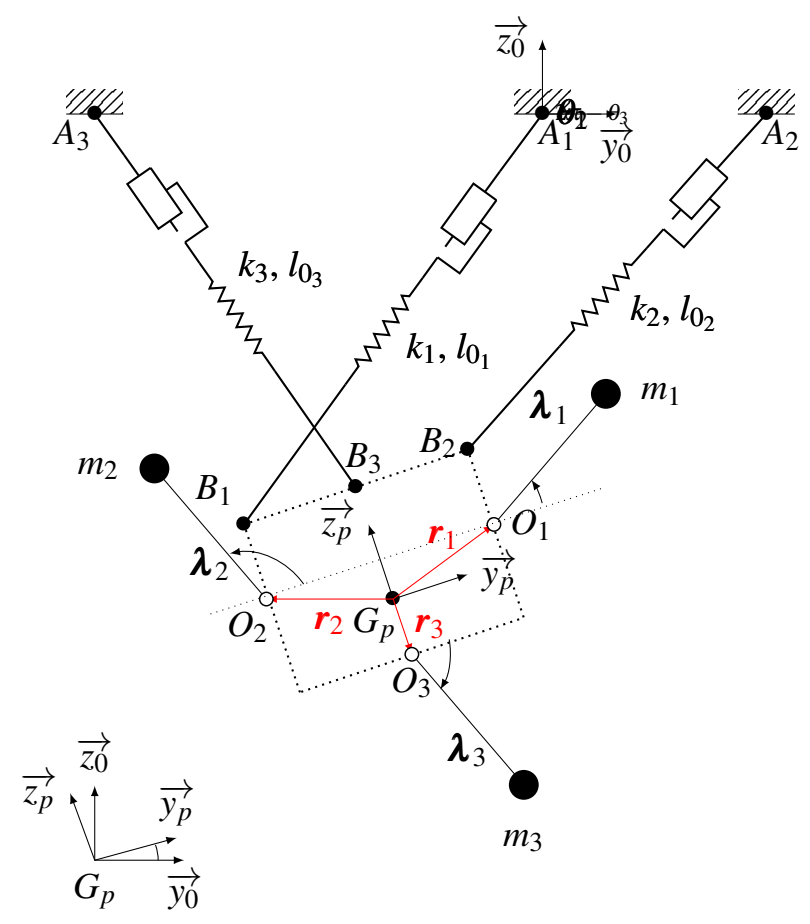

Fig. 2. Schematic of a planar 3-DOF CDPR with 3 stabilization arms

In this paper, a multi-DOF active stabilizer following the principle described above, is placed on-board the mobile platform of a CDPR. The CDPR mobile platform is driven by means of $N$ cables which exit a stationary base frame at points $A_{i}$ and are attached to the CDPR mobile platform at points $B_{i}, i$ being the cable index. The cable lengths are controlled by winches fixed to the base frame. The stabilizer consists of $J$ actuated arms, mounted on the CDPR mobile platform in order to damp its vibrations. The stabilizer is composed of actuated rotating arms terminated by point masses. The rotating arms are identical and attached to the CDPR mobile platform at point $O_{j}, j \in[1 . . J]$, whose position relative to the platform center of mass $G_{p}$ is defined by vector $\boldsymbol{r}_{j}=\overrightarrow{G_{p} O_{j}}$. Each arm length is denoted $\lambda_{j}$ and the point mass is denoted $m_{j}$. The CDPR mobile platform has $n$ DOFs and, in this paper, $J \geq n$.

The $j^{\text {th }}$ arm makes an angle $\theta_{j}$ with respect to a nominal resting position and its actuator provides a torque $\kappa_{j}$. An appropriate nominal resting position of the arms should be chosen so as to maximize the wrench applied by the stabilizer on the CDPR platform. $\boldsymbol{\kappa}$ and $\boldsymbol{\theta}$ are the vectors of motor torques and angle from rest position of the stabilizer arms, respectively.

A planar 3-DOF CDPR with a three-arm stabilizer is shown in Fig. 2. This stabilizer can compensate the vibrations on the 3 DOFs of the planar CDPR mobile platform. In this example, the motors located at points $O_{1}, O_{2}$, and $O_{3}$ are rigidly fixed to the CDPR mobile platform.

\section{DYNAMIC MODELING}

Since the stabilizer is placed on-board the CDPR platform and both the cable forces and the stabilizer movements 
affect the same DOFs in Cartesian space, their dynamics are coupled as detailed in the next section.

\section{A. Static modeling of the CDPR mobile platform}

The frame attached to the CDPR mobile platform is denoted $\mathscr{R}_{p}$ and the fixed base frame is denoted $\mathscr{R}_{0}$. The pose of the platform is given by the vector $\boldsymbol{x}$, composed of the position of the platform center of mass in $\mathscr{R}_{0}$ and of three Euler angles defining the orientation of $\mathscr{R}_{p}$ in $\mathscr{R}_{0}$.

Similarly to [9], [14], the cables are assumed to be massless and all tensed, which allows to neglect the sagging effect [29]. Their longitudinal elasticity is taken into account by modeling them as linear axial springs. The cable tension vector is denoted $\boldsymbol{\tau}$. By means of inverse kinematics, the pose $\boldsymbol{x}$ of the mobile platform gives directly the cable actual (strained) lengths $\boldsymbol{l}_{\mathbf{2}}(\boldsymbol{x})$. The vector of cable unstrained lengths is denoted $\boldsymbol{l}_{\mathbf{1}}$. The cable tensions can then be written as follows:

$$
\boldsymbol{\tau}=k_{c} \operatorname{diag}\left(\boldsymbol{l}_{\mathbf{1}}\right)^{-1}\left(\boldsymbol{l}_{\mathbf{2}}(\boldsymbol{x})-\boldsymbol{l}_{\mathbf{1}}\right)
$$

where $k_{c}$ is the specific axial stiffness per unit of cable length, under the assumption that the $N$ cables have the same constant stiffness.

\section{B. Dynamic modeling of the CDPR with embedded stabilizer}

The dynamics of the stabilizer and the CDPR mobile platform are coupled. The corresponding dynamic model can be obtained using Newton-Euler equations:

$$
\boldsymbol{M}(\boldsymbol{x}, \boldsymbol{\theta})\left(\begin{array}{c}
\ddot{\boldsymbol{x}} \\
\ddot{\boldsymbol{\theta}}
\end{array}\right)+\boldsymbol{C}(\boldsymbol{x}, \dot{\boldsymbol{x}}, \boldsymbol{\theta}, \dot{\boldsymbol{\theta}})\left(\begin{array}{l}
\dot{\boldsymbol{x}} \\
\dot{\boldsymbol{\theta}}
\end{array}\right)+\boldsymbol{G}(\boldsymbol{x}, \boldsymbol{\theta})=\left(\begin{array}{c}
-\boldsymbol{J}^{T} \boldsymbol{\tau} \\
\boldsymbol{\kappa}
\end{array}\right)
$$

where $\boldsymbol{M}$ is the generalized mass matrix of the CDPR mobile platform and its on-board stabilizer, $\boldsymbol{C}$ the Coriolis matrix, $\boldsymbol{G}$ the wrench due to the weight of the whole system (platform and stabilizer) and $\boldsymbol{J}$ the Jacobian matrix of the CDPR. $n$ being the number of DOFs of the CDPR mobile platform, $\boldsymbol{M}$ and $\boldsymbol{C}$ are two square matrices of dimension $(n+J) \times(n+J)$, $\boldsymbol{G}$ is a vector of dimension $(n+J), \boldsymbol{J}$ is a $N \times n$ matrix, $\boldsymbol{\tau}$ a vector of dimension $N$, and $\boldsymbol{\kappa}$ a vector of dimension $J$.

The symmetric positive definite matrix $\boldsymbol{M}$ can be written under the following form:

$$
M=\left[\begin{array}{l|l}
M_{11} & M_{12} \\
\hline M_{12}^{T} & M_{22}
\end{array}\right]
$$

where $\boldsymbol{M}_{11}$ is a square $n \times n$ matrix and $\boldsymbol{M}_{22}$ a $J \times J$ matrix. The matrix $\boldsymbol{C}$ can be partitioned into two submatrices $\boldsymbol{C}_{\mathbf{1}}$ and $\boldsymbol{C}_{\mathbf{2}}$. Likewise, the vector $\boldsymbol{G}$ can be separated into $\boldsymbol{G}_{\mathbf{1}}$ and $\boldsymbol{G}_{\mathbf{2}}$. With these notations, (2) is equivalent to:

$$
\left\{\begin{array}{cl}
M_{11} \ddot{x}+M_{12} \ddot{\theta}+C_{1}(x, \dot{x}, \boldsymbol{\theta}, \dot{\theta}) \dot{x}+G_{1}(x) & =-J^{T} \tau \\
M_{12}{ }^{T} \ddot{x}+M_{22} \ddot{\theta}+C_{2}(x, \dot{x}, \boldsymbol{\theta}, \dot{\theta}) \dot{\theta}+G_{2}(\theta) & =\kappa
\end{array}\right.
$$

\section{DESIGN OF THE CONTROLLER AND STABILITY PROOF}

\section{A. Design of the controller}

The proposed strategy to control the whole system, consisting of the CDPR and the on-board stabilizer, is shown

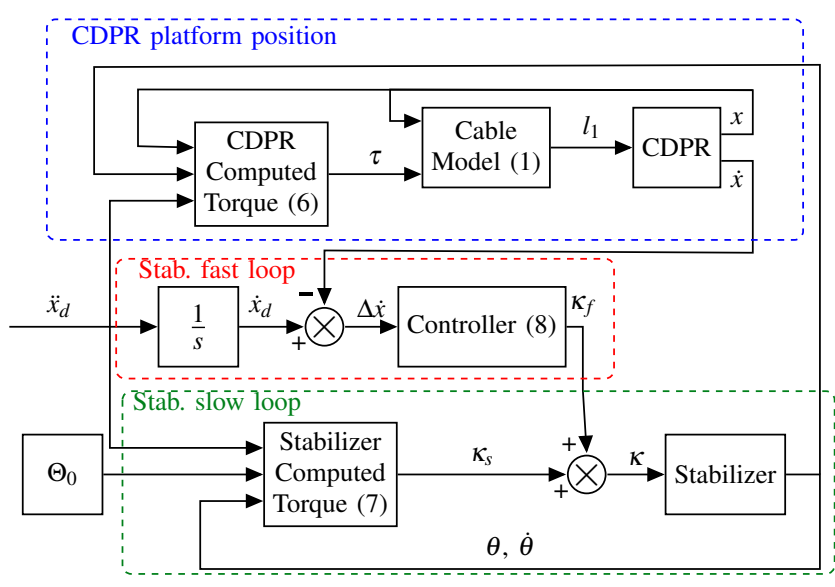

Fig. 3. Control scheme

in Fig. 3. This control scheme is composed of three control loops, two for the stabilizer control and one for the CDPR mobile platform control.

The first stabilizer control loop aims at canceling the CDPR mobile platform vibrations. Since the wrench applied by the stabilizer on the CDPR platform is maximal when the stabilizer arms are at their resting nominal positions, a second stabilizer control loop should be used to bring back the stabilizer arms to their nominal positions to enhance the system performance. The corresponding nominal state is defined as $\Theta_{0}=\left\{\ddot{\boldsymbol{\theta}}_{d}=0, \dot{\boldsymbol{\theta}}_{d}=0, \boldsymbol{\theta}_{d}=0\right\}$ where $\boldsymbol{\theta}_{d}=0$ corresponds to the arm nominal positions. The first loop needs to be fast to be able to compensate vibrations whereas the second loop has to be designed to be much slower than the first one to not interfere with it. In Fig. 3, the first loop is thus called the stabilizer fast loop (shown in red) whereas the second loop is called the slow loop (shown in green). Moreover, as shown in blue in Fig. 3, an inverse dynamics control loop is used to control the CDPR mobile platform position.

These three control loops can be divided into two slow loops, one for the position control of the CDPR platform and one for steering the stabilizer arms back to their nominal positions, and a faster loop for active CDPR vibration damping by means of stabilizer arm fast movements. Because these loops work at different time scales, the singular perturbation theory can be used to prove the stability of the whole system [26]. The use of the singular perturbation theory requires the closed-loop system equations to be split into the two parts. Following the methods described in [25], [30], the system closed-loop equations are given hereafter.

Since the generalized mass matrix $\boldsymbol{M}$ is invertible by definition, let $\boldsymbol{H}$ be its block partitioned inverse:

$$
H=\left[\begin{array}{l|l}
M_{11} & M_{12} \\
\hline M_{12}^{T} & M_{22}
\end{array}\right]^{-1}=\left[\begin{array}{l|l}
H_{11} & H_{12} \\
\hline H_{21} & H_{22}
\end{array}\right]
$$

where $\boldsymbol{H}_{11}$ a $n \times n$ matrix, $\boldsymbol{H}_{12}$ a $n \times J$ matrix, and $\boldsymbol{H}_{22}$ a $J \times J$ matrix. To ensure tracking of a desired platform pose, a nonlinear feedback linearization control loop is set up as 
follows:

$$
\tau=-\left(J^{T}\right)^{+}\left(M_{11} \ddot{x}_{d}+C_{1}(x, \dot{x}, \theta, \dot{\theta}) \dot{x}+G_{1}(x)+H_{11}^{-1}\left(K_{v} \Delta \dot{x}+K_{p} \Delta x\right)\right)
$$

where $\boldsymbol{K}_{\boldsymbol{p}}$ and $\boldsymbol{K}_{\boldsymbol{v}}$ are two positive diagonal high gain matrices and $\boldsymbol{H}_{11}^{-1}$ is used for later simplifications in (13). The subscript $d$ indicates the desired value for a variable and $\Delta \boldsymbol{x}=\boldsymbol{x}_{d}-\boldsymbol{x}$ is the pose error.

For the second subsystem (4b), a composite control law $\boldsymbol{\kappa}=\boldsymbol{\kappa}_{\boldsymbol{s}}+\boldsymbol{\kappa}_{\boldsymbol{f}}$ is proposed for the torque control of the stabilizing arms. First, $\boldsymbol{\kappa}_{\boldsymbol{f}}$ aims at damping the CDPR vibrations through the inertial reaction wrenches of the arms on the CDPR platform:

$$
\kappa_{f}=H_{12}^{+} K_{\eta} \Delta \dot{x}
$$

where $\boldsymbol{K}_{\boldsymbol{\eta}}$ is a positive diagonal gain matrix and $\boldsymbol{H}_{12}^{+}$is the pseudo-inverse of $\boldsymbol{H}_{\mathbf{1 2}}$, useful for later simplifications in (12). Note that, since the case $J \geq n$-at least as many stabilizer arms as number of CDPR platform DOFs-is dealt with in this paper, and under the assumption that no pair of stabilizer arms are aligned, the matrix $\boldsymbol{M}_{\mathbf{1 2}}$ has full row rank and $\boldsymbol{H}_{12}^{+} \boldsymbol{H}_{12}=\boldsymbol{I}_{\boldsymbol{n} \times \boldsymbol{n}}$. Note also that matrices $\boldsymbol{M}_{12}$ and $\boldsymbol{H}_{12}$ depend on time dependent variables $\boldsymbol{\Delta} \boldsymbol{x}$ and $\boldsymbol{\theta}$.

Second, as any wrench can be created on the platform by the stabilizer arms as long as no pair of arms are aligned, $\boldsymbol{\kappa}_{\boldsymbol{s}}$ aims at driving the stabilizer arms back to their resting nominal positions $\Theta_{0}$. Hence, $\boldsymbol{\kappa}_{\boldsymbol{s}}$ is set using the following inverse dynamics approach:

$$
\boldsymbol{\kappa}_{\boldsymbol{s}}=\boldsymbol{M}_{12}^{T} \ddot{\boldsymbol{x}}_{d}+\boldsymbol{C}_{2}(\boldsymbol{x}, \dot{\boldsymbol{x}}, \boldsymbol{\theta}, \dot{\boldsymbol{\theta}}) \dot{\boldsymbol{\theta}}+\boldsymbol{G}_{2}(\boldsymbol{\theta})-\boldsymbol{K}_{\boldsymbol{\omega}} \dot{\boldsymbol{\theta}}-\boldsymbol{K}_{\boldsymbol{\theta}} \boldsymbol{\theta}
$$

where $\boldsymbol{K}_{\boldsymbol{\theta}}$ and $\boldsymbol{K}_{\boldsymbol{\omega}}$ are two positive diagonal gain matrices of an order of magnitude lower than $\boldsymbol{K}_{\boldsymbol{\eta}}$.

By substituting equations (6), (8) and (7) in (4), the nonlinear closed-loop system can be written as follows:

$$
\begin{cases}M_{11} \Delta \ddot{x}-M_{12} \ddot{\theta}+H_{11}^{-1} K_{v} \Delta \dot{x}+H_{11}^{-1} K_{p} \Delta x & =0 \\ M_{12}^{T} \Delta \ddot{x}-M_{22} \ddot{\theta}+H_{12}^{+} K_{\eta} \Delta \dot{x}-K_{\omega} \dot{\theta}-K_{\theta} \theta & =0\end{cases}
$$

The matrices $\boldsymbol{M}$ and $\boldsymbol{H}$ are time dependent through the variables $\boldsymbol{x}_{d}, \boldsymbol{\Delta x}$ and $\boldsymbol{\theta}$.

\section{B. Time scale separation and singular perturbation model}

To retrieve a standard form of the closed-loop system (9) convenient for its stability analysis, it is rewritten under the following matrix representation:

$$
\begin{gathered}
{\left[\begin{array}{ll}
M_{11} & M_{12} \\
M_{12}^{T} & M_{22}
\end{array}\right]\left[\begin{array}{c}
\Delta \ddot{x} \\
-\ddot{\theta}
\end{array}\right]+\left[\begin{array}{cc}
H_{11}^{-1} K_{v} & 0 \\
H_{12}^{+} K_{\eta} & K_{\omega}
\end{array}\right]\left[\begin{array}{c}
\Delta \dot{x} \\
-\dot{\theta}
\end{array}\right]+} \\
{\left[\begin{array}{cc}
H_{11}^{-1} K_{p} & 0 \\
0 & K_{\theta}
\end{array}\right]\left[\begin{array}{c}
\Delta x \\
-\theta
\end{array}\right]=0}
\end{gathered}
$$

and multiplied by $\boldsymbol{M}^{-1}=\boldsymbol{H}$ :

$$
\left\{\begin{aligned}
\Delta \ddot{x} & = & -K_{v} \Delta \dot{x}-K_{\eta} \Delta \dot{x}-K_{p} \Delta x+H_{12}\left(K_{\omega} \dot{\theta}+K_{\theta} \theta\right) \\
\ddot{\theta} & = & H_{12}^{T} H_{11}^{-1} K_{\boldsymbol{v}} \Delta \dot{x}+H_{22} H_{12}^{+} K_{\eta} \Delta \dot{x} \\
& + & H_{12}^{T} H_{11}^{-1} K_{p} \Delta x-H_{22}\left(K_{\boldsymbol{\omega}} \dot{\theta}+K_{\theta} \theta\right)
\end{aligned}\right.
$$

With the assumptions of Section IV-A on the magnitudes of the controller feedback gains, it can be assumed that
$\boldsymbol{K}_{\boldsymbol{\theta}}$ and $\boldsymbol{K}_{\boldsymbol{\omega}}$ are $\mathscr{O}(1)$ gain matrices and that $\boldsymbol{K}_{\boldsymbol{p}}$ is a high gain feedback matrix of order $\mathscr{O}\left(\frac{1}{\varepsilon}\right)$, with $\varepsilon$ a small scalar parameter. Let $\boldsymbol{K}_{\boldsymbol{p}}=\frac{1}{\varepsilon} \boldsymbol{I}_{n \times n}$ and let $\tilde{\boldsymbol{K}}_{\boldsymbol{v}}=\sqrt{\varepsilon} \boldsymbol{K}_{\boldsymbol{v}}$ and $\tilde{\boldsymbol{K}_{\boldsymbol{\eta}}}=$ $\sqrt{\varepsilon} \boldsymbol{K}_{\boldsymbol{\eta}}$ be $\mathscr{O}(1)$ diagonal gain matrices.

Introducing the variable $\boldsymbol{z}=\boldsymbol{K}_{\boldsymbol{p}} \boldsymbol{\Delta x}$, the system (11) can be written under the standard singular perturbation model [25]:

$$
\left\{\begin{aligned}
\varepsilon \ddot{z} & =-\sqrt{\varepsilon}\left(\tilde{\boldsymbol{K}_{\boldsymbol{v}}}+\tilde{\boldsymbol{K}_{\eta}}\right) \dot{\boldsymbol{z}}-\boldsymbol{z}+\boldsymbol{H}_{\mathbf{1 2}}\left(\boldsymbol{K}_{\boldsymbol{\omega}} \dot{\boldsymbol{\theta}}+\boldsymbol{K}_{\boldsymbol{\theta}} \boldsymbol{\theta}\right) \\
\ddot{\boldsymbol{\theta}} & =\sqrt{\varepsilon} \boldsymbol{H}_{21} \boldsymbol{H}_{11}^{-1} \tilde{\boldsymbol{K}_{\boldsymbol{v}}} \dot{\boldsymbol{z}}+\sqrt{\varepsilon} \boldsymbol{H}_{\mathbf{2} 2} \boldsymbol{H}_{\mathbf{1 2}}^{+} \tilde{\boldsymbol{K}_{\eta}} \dot{\boldsymbol{z}}+\boldsymbol{H}_{\mathbf{2 1}} \boldsymbol{H}_{\mathbf{1 1}}^{-1} \boldsymbol{z} \\
& -\boldsymbol{H}_{\mathbf{2 2}}\left(\boldsymbol{K}_{\boldsymbol{\omega}} \dot{\boldsymbol{\theta}}+\boldsymbol{K}_{\boldsymbol{\theta}} \boldsymbol{\theta}\right)
\end{aligned}\right.
$$

For $\varepsilon$ small enough, this system exhibits a two-time scale behavior between a variable $\boldsymbol{\theta}$ varying slowly and $z$ a fast transient variable superimposed on the slowly varying quasisteady state $(\varepsilon \rightarrow 0$ and $\|\ddot{z}\|>>\|\ddot{\theta}\|)$.

Using results from singular perturbation theory derived from Tikhonov's Theorem [23], [30], the stability of the system (12) can then be inferred if both its quasi-steady state (slow) model and its boundary layer (fast transient response) model are stable.

\section{Quasi-steady state model (slow subsystem)}

The quasi-steady state model is derived from the singular perturbation model by setting $\varepsilon=0$. Dividing (12a) by $\varepsilon$ on both sides highlights that $\ddot{z}$ becomes very large when $\varepsilon \rightarrow 0$. Hence, it can be assumed that the variable $z$ converges instantaneously to $z_{s}$, the solution of equation (12a) when $\varepsilon=0$. Moreover, substituting $z$ for $z_{s}$ in (12b) with $\varepsilon=0$ yields the linear quasi-steady state (slow) model for system (12):

$$
\left\{\begin{array}{l}
z_{s}=\boldsymbol{H}_{\mathbf{1 2}}\left(\boldsymbol{K}_{\boldsymbol{\omega}} \dot{\boldsymbol{\theta}}+\boldsymbol{K}_{\boldsymbol{\theta}} \boldsymbol{\theta}\right) \\
\ddot{\boldsymbol{\theta}}=\underbrace{\left(\boldsymbol{H}_{\mathbf{2 2}}-\boldsymbol{H}_{\mathbf{2 1}} \boldsymbol{H}_{\mathbf{1 1}}^{-1} \boldsymbol{H}_{\mathbf{1 2}}\right)}_{\boldsymbol{M}_{\mathbf{2 2}}^{-1}}\left(-\boldsymbol{K}_{\boldsymbol{\omega}} \dot{\boldsymbol{\theta}}-\boldsymbol{K}_{\boldsymbol{\theta}} \boldsymbol{\theta}\right)
\end{array}\right.
$$

The stability of this quasi-steady state model can be proved with the following Lyapunov function based on its kinetic energy and its elastic potential energy:

$$
V_{s}=\frac{1}{2} \dot{\boldsymbol{\theta}}^{T} \boldsymbol{M}_{\mathbf{2 2}} \dot{\boldsymbol{\theta}}+\frac{1}{2} \boldsymbol{\theta}^{T} \boldsymbol{K}_{\boldsymbol{\theta}} \boldsymbol{\theta}
$$

where $\boldsymbol{M}_{\mathbf{2 2}}$ and $\boldsymbol{K}_{\boldsymbol{\theta}}$ are constant positive definite matrices. With relation (13b), its time derivative $\dot{V}_{s}$ is given by:

$$
\dot{V}_{s}=-\dot{\boldsymbol{\theta}}^{T} \boldsymbol{K}_{\boldsymbol{\omega}} \dot{\boldsymbol{\theta}}
$$

Since $\boldsymbol{K}_{\boldsymbol{\omega}}$ is positive definite, $\dot{V}_{s}<0$ for $\dot{\boldsymbol{\theta}} \neq \mathbf{0}$ and, consequently, the origin of the quasi-steady state or reduced linear system is exponentially stable.

\section{Boundary layer model (fast subsystem)}

The quasi-steady state model assumes fast convergence of $z$ to $z_{s}$. This convergence thus requires some stability conditions on this fast dynamics. To study the behavior of the fast dynamics of $z$ with respect to its quasi-steady state solution $z_{s}$, two tools are introduced:

- A change of variable $z_{f}=\boldsymbol{z}-\boldsymbol{z}_{s}$, where $z_{f}$ is the deviation from the quasi-steady state solution $z$; 


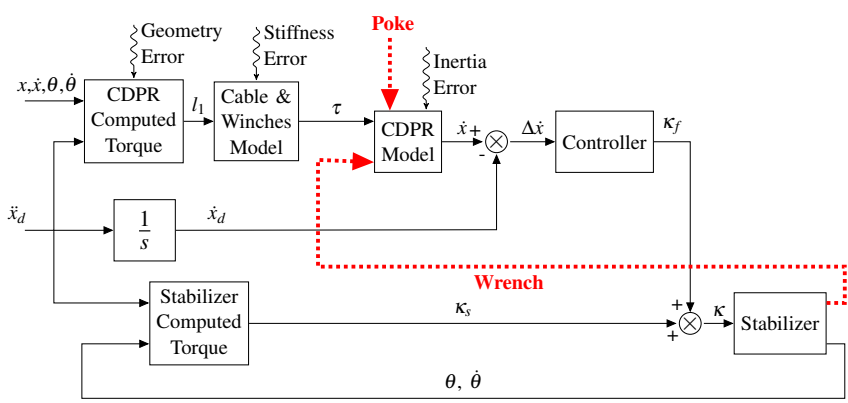

Fig. 4. Simulation schematics, with perturbations added

- A scaled time $\bar{t}=\frac{t}{\sqrt{\varepsilon}}$, where the prime superscript corresponds to a differentiation with respect to $\bar{t}$.

The boundary layer system (12) can be written as:

$$
\left\{\begin{aligned}
z^{\prime \prime}= & -\left(\tilde{\boldsymbol{K}_{\boldsymbol{v}}}+\tilde{\boldsymbol{K}_{\eta}}\right) z^{\prime}-z+\boldsymbol{H}_{\mathbf{1 2}}\left(\frac{1}{\sqrt{\varepsilon}} \boldsymbol{K}_{\boldsymbol{\omega}} \boldsymbol{\theta}^{\prime}+\boldsymbol{K}_{\boldsymbol{\theta}} \boldsymbol{\theta}\right) \\
\boldsymbol{\theta}^{\prime \prime} & =\varepsilon\left(\boldsymbol{H}_{\mathbf{2 1}} \boldsymbol{H}_{\mathbf{1 1}}^{-1} \tilde{\boldsymbol{K}_{\boldsymbol{v}}} z^{\prime}+\boldsymbol{H}_{\mathbf{2 2}} \boldsymbol{H}_{\mathbf{1 2}}{ }^{-1} \tilde{\boldsymbol{K}_{\eta}} z^{\prime}+\boldsymbol{H}_{\mathbf{2 1}} \boldsymbol{H}_{\mathbf{1 1}}^{-\mathbf{1}} z\right. \\
& \left.-\boldsymbol{H}_{\mathbf{2 2}}\left(\frac{1}{\sqrt{\varepsilon}} \boldsymbol{K}_{\boldsymbol{\omega}} \boldsymbol{\theta}^{\prime}+\boldsymbol{K}_{\boldsymbol{\theta}} \boldsymbol{\theta}\right)\right)
\end{aligned}\right.
$$

When $\varepsilon \rightarrow 0$, the fast time scale $\bar{t} \rightarrow \infty$ and thus time is stretched out. On the one hand, the "slow" variable $\boldsymbol{\theta}$ appears to be frozen in this fast time scale for $\varepsilon=0$. On the other hand, the fast model for the variable $z_{f}$ is given by:

$$
\boldsymbol{z}_{f}^{\prime \prime}=-\left(\tilde{\boldsymbol{K}_{\boldsymbol{v}}}+\tilde{\boldsymbol{K}_{\boldsymbol{\eta}}}\right) \boldsymbol{z}_{f}^{\prime}-\boldsymbol{z}_{f}
$$

As previously, the following Lyapunov function based on the kinetic energy and elastic (unitary stiffness) potential energy can be selected to prove the stability of the boundary layer system:

$$
V_{f}=\frac{1}{2} z_{f}^{\prime T} z_{f}^{\prime}+\frac{1}{2} z_{f}^{T} z_{f}
$$

According to Eq. (17), the stretched time derivative $V_{f}^{\prime}$ is:

$$
V_{f}^{\prime}=\boldsymbol{z}_{f}^{\prime T} \boldsymbol{z}_{f}^{\prime \prime}+\boldsymbol{z}_{f}^{\prime T} \boldsymbol{z}_{f}=-\boldsymbol{z}_{f}^{\prime T}\left(\tilde{\boldsymbol{K}_{v}}+\tilde{\boldsymbol{K}_{\eta}}\right) \boldsymbol{z}_{f}^{\prime}
$$

where $\tilde{\boldsymbol{K}_{\eta}}$ and $\tilde{\boldsymbol{K}_{v}}$ are positive definite. Since $V_{f}^{\prime}<0, \forall \boldsymbol{z}_{f}^{\prime} \neq$ $\mathbf{0}$, the origin $\left\{z_{f}, z_{f}^{\prime}\right\}=\{\mathbf{0}, \mathbf{0}\}$ of the linear boundary layer system is exponentially stable.

As the quasi-steady state (slow) model and the boundary layer (fast) model are both exponentially stable, the theorem 11.4 from [30] states that, for an $\varepsilon$ small enough, the system (12) is also exponentially stable at the origin.

\section{SIMULATION RESULTS}

This section presents the simulations performed in order to evaluate the control approach designed for the CDPR embedding the stabilizer introduced in Section II. First, the simulation model is introduced, then the simulation results are presented, and the influence of various parametric errors

\begin{tabular}{|c|c|}
\hline Parameter & Value \\
\hline \multirow{2}{*}{$\mathscr{R}_{0} \boldsymbol{A}$} & $\left.\begin{array}{lll}0 & 0.24 & -2\end{array}\right]$ \\
\hline & $0 \quad 0$ \\
\hline \multirow{2}{*}{${ }^{\mathscr{R}_{p}} \boldsymbol{B}$} & $\begin{array}{lll}-0.12 & 0.12 & 0\end{array}$ \\
\hline & $\begin{array}{lll}0.15 & 0.15 & 0.15\end{array}$ \\
\hline$k_{c}$ & $500 \mathrm{Nm}^{-1}$ \\
\hline$x_{0}$ & {$\left[\begin{array}{lll}-0.94 \mathrm{~m} & -1 \mathrm{~m} & 0 \mathrm{rad}\end{array}\right]$} \\
\hline $\boldsymbol{\theta}_{0}$ & {$\left[\begin{array}{lll}0 & \pi & \frac{-\pi}{2}\end{array}\right]^{T}$} \\
\hline$m_{p}$ & $3.2 \mathrm{~kg}$ \\
\hline$\lambda_{j}$ & $0.1 \mathrm{~m}$ \\
\hline$m_{j}$ & $0.15 \mathrm{~kg}$ \\
\hline
\end{tabular}
is finally discussed.
TABLE I

MODEL PARAMETER VALUES

\section{A. Presentation of the simulation model}

In the simulations, the planar 3-DOF suspended CDPR with an on-board stabilizer composed of three rotating arms, as shown in Fig. 2, is considered.

The simulation model of this robot has been implemented in Matlab-Simulink, with the goal of using the same overall structure for the current simulation and for later experimental studies using embedded Simulink functionalities. The physical behavior of the CDPR and the stabilizer are simulated using a Simscape Multibody model. Simscape Multibody is a Matlab Simulink toolbox solving the equations of motion for a multibody mechanical system.

The CDPR is chosen to be a planar 3-DOF cablesuspended robot driven by 3 cables. This CDPR being suspended, the gravity passively ensures that the cables remain in tension. The values of the geometric parameters of this CDPR have been chosen close to the ones in [31], with the distances $A_{1} A_{2}=B_{1} B_{2}$. As shown in Fig. 2, the vertices of the mobile platform upper edge are attached to the points $A_{1}$ and $A_{2}$. The position of the attachment point $A_{3}$ has been selected so as to maximize the size of the Wrench Feasible Workspace (WFW) [32]. The cable $A_{3} B_{3}$ is such that point $A_{3}$ is positioned at the limit of the workspace and $B_{3}$ at the center of the platform upper edge. The initial position of the mobile platform is denoted $\boldsymbol{x}_{0}$.

The stabilizer mounted on-board the suspended CDPR mobile platform consists of three rotating arms. In the current model, the arms are represented by massless bars of length $\lambda_{j}$ having point masses $m_{j}$ attached at their ends. The resting nominal (initial) configuration of the arms allows a good clearance without collision with the CDPR platform and cables, and allows the application of forces along the $\mathrm{Y}$ and $\mathrm{Z}$ axes and moments along the out-of-the-plane $\mathrm{X}$ axis. In the present study, the initial arm position $\boldsymbol{\theta}_{0}$ was chosen to maximize the stroke for each arm. During the simulations, no collision between the stabilizer arms and the cables has been observed.

The values of the parameters used in the simulations are given in Tab. I. 
TABLE II

Control Gains

\begin{tabular}{|c|l|}
\hline Coefficient & Value \\
\hline $\boldsymbol{K}_{\boldsymbol{p}}$ & $500 \operatorname{diag}\left(\left[\begin{array}{lll}1 & 1 & 1\end{array}\right]\right)$ \\
$\boldsymbol{K}_{\boldsymbol{v}}$ & $5 \operatorname{diag}\left(\left[\begin{array}{lll}1 & 1 & 1\end{array}\right]\right)$ \\
$\boldsymbol{K}_{\boldsymbol{\theta}}$ & $2.10^{-2} \operatorname{diag}\left(\left[\begin{array}{lll}1 & 1 & 1\end{array}\right]\right)$ \\
$\boldsymbol{K}_{\boldsymbol{\omega}}$ & $5.10^{-2} \operatorname{diag}\left(\left[\begin{array}{lll}1 & 1 & 1\end{array}\right]\right)$ \\
$\boldsymbol{K}_{\boldsymbol{\eta}}$ & $250 \operatorname{diag}\left(\left[\begin{array}{lll}0.8 & 0.5 & 0.95\end{array}\right]\right)$ \\
\hline
\end{tabular}

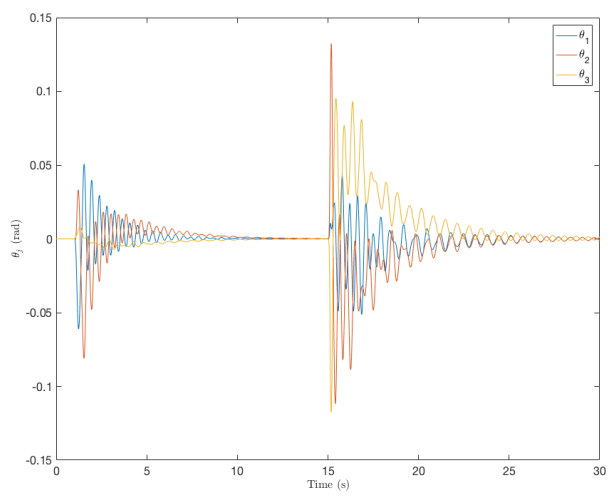

Fig. 5. Positions of the stabilizer arms

\section{B. Simulation results}

The control scheme implemented in the Simulink simulation model is the one of Fig. 3 and introduced in Section IV. The vibration speed is obtained by subtracting the "measured" speed to the desired speed $\dot{\boldsymbol{x}}_{d}$ obtained by integrating the desired acceleration $\ddot{x}_{d}$. The structure of the simulation is presented in Fig. 4. In this figure, the various parametric error sources discussed later in Section V-C are shown.

The CDPR platform and the stabilizer are simulated using a Simscape model. The "Cable and Winch Models" block implements, on the one hand, the relationship between the cable tension and its unstrained length from Eq. (1), and, on the other hand, the low-level control of the winches. The winch motor are servoed with a RST polynomial control law. The actuators of the stabilizer arms are also controlled in position with a classic PID control.

The gain values used in the three control loops of Fig. 3 are given in Tab. II. These gains have been tuned to be effective close to the center of the WFW of the CDPR.

The simulation experiment starts at the center of the WFW at $\boldsymbol{x}_{\mathbf{0}}=\left[\begin{array}{llll}-0.94 \mathrm{~m} & -1 \mathrm{~m} & 0 \mathrm{rad}\end{array}\right]^{T}$. First a step motion of $\left[\begin{array}{llll}0 \mathrm{~m} & 0.2 \mathrm{~m} & 0 \mathrm{rad}\end{array}\right]^{T}$ along the vertical axis is performed. After 15 seconds it is perturbed with a vertical a poke (a short force input of $20 \mathrm{~N}$ along the vertical axis applied to the CDPR platform). In both cases, the action creates a perturbation wrench putting the robot into a vibrating motion, mostly along the $\mathrm{Z}$ axis.

To compensate these vibrations, the stabilizer arms move to produce a wrench opposed to the perturbation, by means

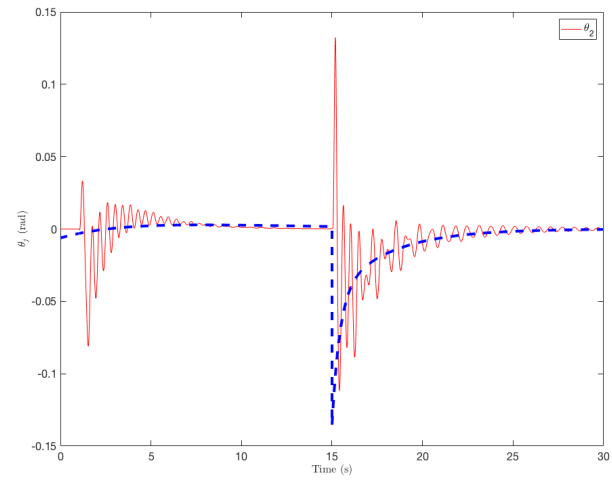

Fig. 6. Position of arm 2

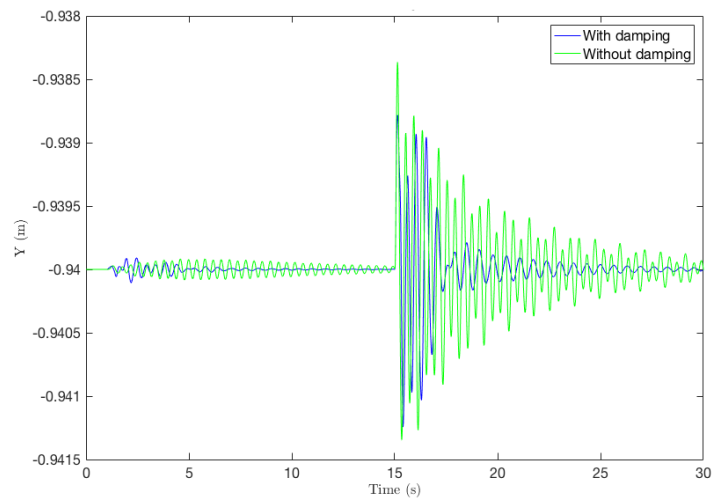

Fig. 7. Damping along the $Y$ axis

of the control law defined in Section IV. The movements of the three arms are shown in Fig. 5. On the curve representing the trajectory of the second arm shown in Fig. 6, it is possible to distinguish the two parts of the control law. In red the fast movement made to compensate the vibrations and in dashed blue the slow one that drives the arm back to its nominal position.

The resulting damped vibrations using the gain values in Tab. II are compared to the free responses, where the stabilizers do not move and only the CDPR inverse dynamics control loop has an effect on the vibrations. With the proposed method using the stabilizer, the vibrations are damped much faster, which can be seen in Figs. 7, 8 and 9, where the green curves correspond to the free responses (without stabilizer) and the blue ones to the damped responses. As shown in Fig. 8, when the stabilizer is used, the vibrations along the $\mathrm{Z}$ axis are damped in $5 s$ whereas, without the stabilizer, the vibrations are damped only by the mobile platform position control loop in $15 s$.

\section{Influence of parametric errors}

In order to evaluate the influence of inaccuracies in the modeling, the parameters most subjected to measurement errors have been identified. On these parameters, an error in a given confidence interval has been added, in order to assess the robustness of the stabilizer, i.e., its efficiency in 


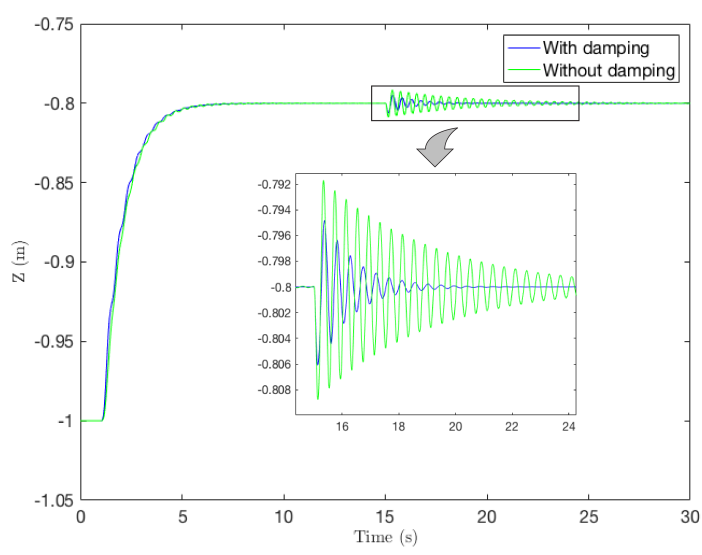

Fig. 8. Damping along the $Z$ axis

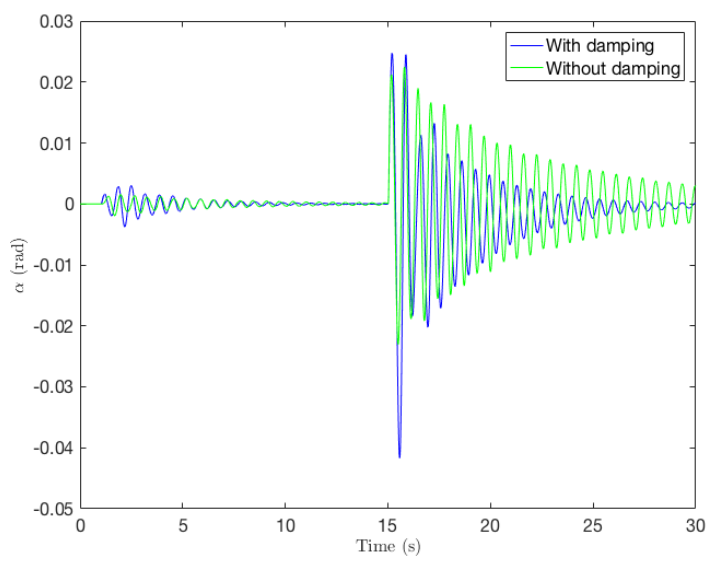

Fig. 9. Damping on the rotation around $X$

rejecting perturbations due to parameter uncertainties.

The uncertain parameters considered in this study are the geometry of the CDPR (the positions of the cable attachment points on the base frame and on the mobile platform), the position of the platform center of mass, and the stiffness of the cables. Simulation experiments similar to the ones in Section V-B but for a step value of $\left[\begin{array}{lllll}0.2 \mathrm{~m} & -0.5 \mathrm{~m} & \frac{\pi}{10} \mathrm{rad}\end{array}\right]^{T}$ have been executed. Each test has been performed with a random error added to all the geometric parameters and to the cable stiffness. These errors are comprised within $\pm 5 \%$ of the position parameter nominal values and $\pm 10 \%$ for the cable stiffness, the nominal values being given in Tab. I. Uniform distribution of these random errors has been considered. The results of these tests are shown in Tab. III where the mean value and standard deviation (std) of a quality index over 200 simulations are given. The Root Mean Square (RMS) errors on the three DOFs $\sigma_{y}, \sigma_{z}$ and $\sigma_{\alpha}$ have been considered as the quality index. The RMS position error $\sigma_{y}$ on the translation along the $Y$ axis is defined as:

$$
\sigma_{y}=\sqrt{\sum_{k=1}^{N_{s}}\left(\hat{y}_{k}-y_{d_{k}}\right)^{T}\left(\hat{y}_{k}-y_{d_{k}}\right)}
$$

where $\hat{y}_{k}$ is the $k^{t h}$ sample of $\hat{y}(t), \hat{y}$ the "measured" value, $y_{d}$ the desired value and $N_{s}$ the number of samples. Similar
TABLE III

INFLUENCE OF PARAMETRIC UNCERTAINTIES ON DAMPING

\begin{tabular}{|l|l|c|c|c|}
\hline \multicolumn{2}{|c|}{ Case } & $\sigma_{y}$ & $\sigma_{z}$ & $\sigma_{\alpha}$ \\
\hline & mean & 0.0256 & 0.1098 & 0.5955 \\
With stabilizer & std & 0.0111 & 0.0914 & 0.4875 \\
\hline & mean & 0.0290 & 0.1180 & 0.7987 \\
Without stabilizer & std & 0.0130 & 0.0927 & 0.5012 \\
\hline
\end{tabular}

TABLE IV

DAMPING QUALITY

\begin{tabular}{|l|c|c|c|}
\hline \multicolumn{1}{|c|}{ Case } & $\sigma_{y}$ & $\sigma_{z}$ & $\sigma_{\alpha}$ \\
\hline With stabilizer & 0.0274 & 0.0525 & 0.6325 \\
\hline Without stabilizer & 0.0298 & 0.0573 & 0.6238 \\
\hline
\end{tabular}

definitions give the RMS position errors $\sigma_{z}$ and $\sigma_{\alpha}$ for the translation along the $Z$ axis and for the rotation around $X$, respectively.

The influence of the parametric errors given in Tab. III are to be compared to the damping quality index in ideal conditions (no parametric uncertainty) shown in Tab. IV. As expected, on overall, the parametric uncertainties are seen to have a non-negligible impact on the position errors. Nevertheless, the embedded stabilizer remains efficient in all these simulation cases.

\section{CONCLUSIONS}

In this paper, an original device for active stabilization of a CDPR has been presented. This device consists of a stabilizer made of actuated rotating arms placed on-board the CDPR mobile platform. A control strategy for active vibration damping of the CDPR platform equipped with the stabilizer has also been proposed. The stability of the corresponding closed-loop system has been analyzed using singular perturbation theory and the Lyapunov's second method. Finally, the efficiency and robustness of the proposed device and of the control strategy have been tested in simulations in the case of a planar 3-DOF CDPR equipped with a stabilizer composed of three rotating arms.

Unnecessary movements of the stabilizer arms may occur using the proposed control strategy because actuation redundancy in the whole system consisting of the CDPR and the stabilizer is resolved by means of pseudo-inverse calculations. Part of the future work will deal with the integration into the control strategy of more efficient redundancy resolution methods.

A planar 3-DOF CDPR demonstrator is currently being built to validate the current approach on a physical test bed. A multi-DOF version of the stabilizer is also being designed, for future use on the spatial 6-DOF CDPR CoGiRo.

\section{ACKNOWLEDGEMENTS}

This work was supported by the ANR under grant ANR15-CE10-0006, project DexterWide.

\section{REFERENCES}

[1] S. Kawamura, W. Choe, S. Tanaka, and S. Pandian, "Development of an ultrahigh speed robot falcon using wire drive system," in IEEE International Conference on Robotics and Automation, 1995. 
[2] C. Lambert, M. Nahon, and D. Chalmers, "Implementation of an aerostat positioning system with cable control," IEEE/ASME Transactions on Mechatronics, vol. 12, no. 1, 2007.

[3] J.-P. Merlet and D. Daney, "A portable, modular parallel wire crane for rescue operations," in IEEE International Conference on Robotics and Automation, 2010.

[4] A. Pott, H. Mutherich, W. Kraus, V. Schmidt, P. Miermeister, and A. Verl, "Ipanema: A family of cable-driven parallel robots for industrial applications," in Cable-Driven Parallel Robots, 2013.

[5] M. Gouttefarde, J.-F. Collard, N. Riehl, and C. Baradat, "Geometry selection of a redundantly actuated cable-suspended parallel robot," IEEE Transactions on Robotics, vol. 31, no. 2, 2015.

[6] L. Gagliardini, S. Caro, M. Gouttefarde, and A. Girin, "Discrete reconfiguration planning for cable-driven parallel robots," Mechanism and Machine Theory, vol. 100, 2016.

[7] S. Kawamura, H. Kino, and C. Won, "High-speed manipulation by using parallel wire-driven robots," Robotica, vol. 18, no. 1, 2000.

[8] S. Behzadipour and A. Khajepour, "Stiffness of cable-based parallel manipulators with application to stability analysis," Journal of $\mathrm{Me}$ chanical Design, vol. 128, no. 1, 2006

[9] X. Weber, L. Cuvillon, and J. Gangloff, "Active vibration canceling of a cable-driven parallel robot using reaction wheels," in IEEE/RSJ International Conference on Intelligent Robots and Systems, 2014.

[10] X. Diao and O. Ma, "Vibration analysis of cable-driven parallel manipulators," Multibody System Dynamics, vol. 21, no. 4, 2009.

[11] M. A. Khosravi and H. D. Taghirad, "Dynamic modeling and control of parallel robots with elastic cables: singular perturbation approach," IEEE Transactions on Robotics, vol. 30, no. 3, 2014.

[12] F. Montgomery and J. Vaughan, "Modeling and control of a cabledriven robot for inspection of wide-area horizontal workspaces,' in Dynamic Systems and Control Conference, American Society of Mechanical Engineers, 2016.

[13] N. C. Singer and W. P. Seering, "Preshaping command inputs to reduce system vibration," Journal of Dynamic Systems, Measurement, and Control, vol. 112, no. 1, 1990.

[14] X. Weber, L. Cuvillon, and J. Gangloff, "Active vibration canceling of a cable-driven parallel robot in modal space," in IEEE International Conference on Robotics and Automation, 2015.

[15] Y. Cheng, G. Ren, and S. Dai, "Vibration control of gough-stewart platform on flexible suspension," IEEE Transactions on Robotics, vol. 19, no. 3, 2003.

[16] S. Lee and W. Book, "Robot vibration control using inertial damping forces," in CISM-IFToMM Symposium Symposium on Robot Design, Dynamics and Control, 1990.

[17] M. Rushton and A. Khajepour, "Optimal actuator placement for vibration control of a planar cable-driven robotic manipulator," in American Control Conference, 2016.

[18] S. Trimpe and R. D'Andrea, "The balancing cube: A dynamic sculpture as test bed for distributed estimation and control," IEEE Control Systems Magazine, vol. 32, no. 6, 2012.

[19] T. Libby, A. M. Johnson, E. Chang-Siu, R. Full, and D. Koditschek, "Comparative design, scaling and control of appendages for inertial reorientation," IEEE Transactions on Robotics, vol. 32, no. 6, 2016.

[20] G. He, X. Tan, X. Zhang, and Z. Lu, "Modeling, motion planning and control of one-legged hopping robot actuated by two arms," Mechanism and Machine Theory, vol. 43, no. 1, 2008.

[21] F. Montgomery and J. Vaughan, "Suppression of cable suspended parallel manipulator vibration utilizing input shaping," in Conference on Control Technology and Applications, 2017.

[22] Rushton, Mitchell, "Vibration control in cable robots using a multiaxis reaction system," Master's thesis, Univ. Waterloo, 2016.

[23] F. Ghorbel, J. Y. Hung, and M. W. Spong, "Adaptive control of flexible joint manipulators," IEEE Control Systems Magazine, vol. 9, no. 7, 1989

[24] J. Lin, Z. Huang, and P. Huang, "An active damping control of robot manipulators with oscillatory bases by singular perturbation approach,' Journal of sound and vibration, 2007.

[25] B. Siciliano and W. J. Book, "A singular perturbation approach to control of lightweight flexible manipulators," International Journal of Robotics Research, vol. 7, no. 4, 1989.

[26] P. V. Kokotovic, R. E. O'Malley Jr, and P. Sannuti, "Singular perturbations and order reduction in control theory - an overview," Automatica vol. 12, no. 2, 1976.

[27] R. Williams, P. Gallina, and J. Vadia, "Planar translational cable-directdriven robots," Journal of Field Robotics, vol. 20, no. 3, 2003.

[28] S.-R. Oh and S. K. Agrawal, "Generation of feasible set points and control of a cable robot," IEEE Transactions on Robotics, vol. 22 no. 3, 2006

[29] N. Riehl, M. Gouttefarde, C. Baradat, and F. Pierrot, "On the determination of cable characteristics for large dimension cable-driven parallel mechanisms," in IEEE International Conference on Robotics and Automation, 2010

[30] H. K. Khalil, Nonlinear Systems. Pearson, 2001.

[31] X. Jiang and C. Gosselin, "Dynamically feasible trajectories for threedof planar cable-suspended parallel robots," in ASME International Design Engineering Technical Conferences and Computers and Information in Engineering Conference, 2014.

[32] M. Gouttefarde, D. Daney, and J.-P. Merlet, "Interval-analysis-based determination of the wrench-feasible workspace of parallel cabledriven robots," IEEE Transactions on Robotics, vol. 27, no. 1, 2011. 